\title{
BOMBEIRO MILITAR E SAÚDE: PRÁTICAS E DESAFIOS - UMA PERSPECTIVA DO GRUPO DE ESTUDOS EM FISIOLOGIA E EPIDEMIOLOGIA DO EXERCÍCIO E DA ATIVIDADE FÍSICA (GEAFS)
}

Luiz Guilherme Grossi Porto ${ }^{1-3}$ Guilherme Eckhardt Molina ${ }^{1,2}$ Daniel Rodrigues Ferreiras Saint Martin ${ }^{2,4}$ Edgard de Melo Keene von Koenig Soares ${ }^{2,4}$ João Paulo Araujo Barbosa ${ }^{2,5}$

Kevin Alves Barreto ${ }^{2,6}$ Carlos Janssen Gomes da Cruz,7

\section{RESUMO}

Rosenkranz Maciel Nogueira ${ }^{2,8}$

Neste dossiê temático, a saúde do bombeiro militar foi abordada na perspectiva das pesquisas conduzidas pelo Grupo de Estudos em Fisiologia e Epidemiologia do Exercício e da Atividade Física (GEAFS), da Faculdade de Educação Física da Universidade de Brasília, que conta com importante parceria na área com o Corpo de Bombeiros Militar do Distrito Federal, desde 2011, e com colaboradores internacionais da Harvard University e do Skidmore College, nos Estados Unidos. Em uma abordagem narrativa, a temática foi analisada em sete grandes tópicos: 1 - as recomendações gerais de atividade física (ATF) para a saúde (tanto em serviço quanto nas folgas); 2 - a importância da aptidão cardiorrespiratória; 3 - a importância do treinamento muscular; 4 - composição corporal e aptidão física; 5 - riscos à saúde associados à atividade profissional e a triagem médico-ocupacional; 6 - o papel do sono na saúde do bombeiro militar e 7 - a função autonômica cardíaca e a frequência cardíaca como indicadores de saúde cardiovascular. Todas as análises foram mediadas pela relação com a aptidão física.

Palavras-chave: Aptidão física; Risco cardiovascular; Atividade Física; Treinamento físico; Saúde.

1: PhD; Professor da Faculdade de Educação Física - Programa de Pós-Graduação em Educação Física; Universidade de Brasília (FEF-UnB); 2: Grupo de Estudos em Fisiologia e Epidemiologia do Exercício e da Atividade Física - FEF-UnB; 3: Harvard T.H. Chan School of Public Health - collaborator; 4: Faculdade de Educação Física - Doutorando do Programa de Pós-Graduação em Educação Física - FEF-UnB; 5 : Faculdade de Educação Física - Mestrando do Programa de Pós-Graduação em Educação Física - FEFUnB; 6: Faculdade de Educação Física - FEF-UnB; aluno de iniciação científica - FAPDF; 7: Centro Universitário Euro-Americano - Departamento de Educação Física 8: Corpo de Bombeiros Militar do Distrito Federal - luizggporto@gmail.com

O presente trabalho foi realizado com apoio de: Coordenação de Aperfeiçoamento de Pessoal de Nível Superior - Brasil (CAPES) - Código de Financiamento 001 - bolsa de doutorado de DRFSM e EMKvKS; b) Fundação de Apoio à Pesquisa do Distrito Federal FAPDF - Edital demanda espontânea 3/2016 (bolsa de IC de $K A B)$. 


\title{
MILITARY FIREFIGHTER AND HEALTH: PRACTICES AND CHALLENGES - A PERSPECTIVE FROM THE STUDY GROUP ON PHYSIOLOGY AND EPIDEMIOLOGY OF EXERCISE AND PHYSICAL ACTIVITY (GEAFS)
}

\begin{abstract}
In this thematic issue, firefighters' health was addressed in the perspective of the researches conducted by the Study Group in Physiology and Epidemiology of Exercise and Physical Activity (GEAFS), from the Faculty of Physical Education of the University of Brasilia, which has an important partnership with the Federal District Firefighter Brigade (CBMDF) and international collaborations from the Harvard University and the Skidmore College, both in the US. On a narrative approach, the thematic was analyzed in 7 big topics: 1 general recommendations of physical activity for health; 2 - the importance of cardiorespiratory fitness; 3 - the importance of muscular training; 4 - body composition and physical fitness; 5 - health-related risks associated with the professional task and the occupational-medical screening; 6 - the role of sleep on military firefighters' health and 7 - the cardiac autonomic function and the recovery heart rate as cardiovascular health proxies. All analyses were mediated by their relationship with physical fitness.
\end{abstract}

Key words: Physical fitness. Cardiovascular risk. Physical Activity. Physical training. Health. 


\section{INTRODUÇÃO}

A edição de dossiê temático sobre a saúde do bombeiro militar parecenos absolutamente apropriada. Sendo a revista Flammae um periódico destinado a divulgação técnico-científica em diversas áreas relevantes à profissão de bombeiro militar, acerta seu corpo editorial em propor um dossiê voltado para a saúde daqueles que realizam as atividades de risco diuturnamente. Há que se cuidar de quem cuida, de quem tem como atribuição precípua o que está definido no lema do Corpo de Bombeiros Militar do Distrito Federal (CBMDF): Vidas alheias e riquezas salvar (CBMDF, 2020).

Desde 2011 desenvolvemos pesquisas com foco na saúde de bombeiros militares em associação com a aptidão física. Assim, muito nos honra participar deste dossiê temático. Está muito bem documentado que a atividade de bombeiro é uma profissão de risco, associada a elevadas taxas de morbimortalidade cardiovascular e metabólica, entre outras (KALES; SMITH, 2017; PORTO, 2018; SOTERIADES et al., 2011). A preocupação com os riscos da profissão não é recente. Há mais de 120 anos nascia umas das mais importantes associações com esta finalidade no mundo: a NFPA - National Fire Protection Association, situada em Quincy, Massachusetts, nos Estados Unidos, tendo como missão ajudar a salvas vidas e reduzir perdas por meio da informação, conhecimento e paixão (NFPA, 2020).

Bombeiros são trabalhadores rotineiramente expostos a diferentes fatores de risco à saúde, como a exposição ao fogo e à fumaça, ao calor excessivo, o estresse físico e emocional. Várias das tarefas ocupacionais inerentes ao dia a dia do bombeiro geram elevada sobrecarga cardiovascular, podendo resultar em condições extremas como a morte súbita cardíaca, doenças cardiovasculares precoces e doenças respiratórias. Essas e outras condições crônicas, como a obesidade e a síndrome metabólica, afetam tanto a saúde quanto a qualidade de vida e a capacidade laboral desses homens e 
mulheres ao longo de suas vidas (GAINEY et al., 2018; KALES; SMITH, 2017; PORTO et al., 2019b; SOTERIADES et al., 2011)

Portanto, é fundamental investigar continuamente os riscos a que bombeiros estão inerentemente expostos em suas jornadas de trabalho, e ao longo de suas carreiras, com vistas ao desenvolvimento de estratégias baseadas em evidências que possam mitigar os riscos e promover a saúde, a qualidade de vida e/ou o desempenho profissional desses trabalhadores. Este artigo será apresentado em 7 grandes tópicos: 1 - as recomendações gerais de atividade física para a saúde; 2 - a importância da aptidão cardiorrespiratória para o bombeiro; 3 - a importância do treinamento muscular; 4 - as relações entre a composição corporal e a aptidão física; 5 - uma visão global dos riscos à saúde do bombeiro, com foco em evidências que subsidiam a triagem médico-ocupacional regular; 6 - o papel do sono na saúde do bombeiro militar e 7 - um olhar mais específico na função cardiovascular com foco na função autonômica cardíaca e no comportamento da frequência cardíaca.

\section{1 - Recomendações gerais de atividade física para a saúde}

A produção de evidências científicas sistematizadas sobre a relação entre atividade física (ATF) e saúde têm seu início em 1953 com o estudo de Morris e colaboradores. Este trabalho demonstrou que os cobradores dos famosos ônibus londrinos de dois andares apresentaram menor chance de desenvolver doenças cardiovasculares quando comparados aos motoristas, que permaneciam sentados (MORRIS et al., 1953). Foram necessários mais 42 anos para a publicação da primeira recomendação oficial de ATF para a saúde (PATE et al., 1995). As recomendações vêm tendo atualizações e em 2018 foi publicada a mais recente diretriz do governo norte-americano reforçando que todo cidadão deve acumular pelo menos 150 minutos/semana de ATF aeróbica de intensidade moderada-vigorosa (MV), ou $75 \mathrm{~min} / \mathrm{sem}$ de ATF vigorosa, e realizar treino de força duas vezes por semana (PHYSICAL ACTIVITY GUIDELINES ADVISORY COMMITTEE, 2018). A despeito das incontáveis pesquisas científicas, os dados demonstram que, infelizmente, as taxas de 
inatividade física estão relativamente estáveis nos últimos anos (GUTHOLD et al., 2018), podendo ser considerada uma pandemia (KOHL et al., 2012).

Em paralelo ao nível de ATF, os estudos têm mostrado que é necessário estarmos atentos também ao excesso de tempo sentado (TS). Evidências sugerem que são necessários de 60 - $75 \mathrm{~min} /$ dia de ATF de intensidade moderada para eliminar o aumento do risco de morte associado ao excesso de TS (EKELUND et al., 2016). Provavelmente essa não é a realidade de boa parte da população, visto que poucos acumulam entre $7 \mathrm{~h}$ e $8 \mathrm{~h}: 45 \mathrm{~min}$ de ATF moderada semanalmente. Estudo desenvolvido por nosso grupo na UnB demonstrou que em um dia de trabalho bombeiros especialistas em combate a incêndio florestal acumularam, em um plantão de 24h, em média 95 min em ATF moderada/vigorosa e 13.147 passos. Entretanto, nos dias de folga esses indicadores foram reduzidos a $34 \mathrm{~min}$ de ATF moderada/vigorosa e 7.431 passos (SAINT MARTIN et al., 2019), indicando a necessidade de melhor compreensão da ATF e do TS nos dias de folga.

As evidências sugerem que a substituição da posição sentada por atividades de intensidade leve (ficar em pé ou caminhar) podem resultar em benefícios a saúde, como melhora da sensibilidade a insulina, da circulação de lipídios e da diminuição da pressão arterial diastólica em indivíduos sedentários, com sobrepeso e obesos (BUMAN et al., 2014; DUVIVIER et al., 2017). Indivíduos com baixo nível de ATF ( $\leq 17 \mathrm{~min} / \mathrm{dia}$ ATF-MV) que substituíram $30 \mathrm{~min} /$ dia na posição sentada por $30 \mathrm{~min} /$ dia de ATF leve apresentaram menos $9 \%$ de risco de morte por câncer e menos $19 \%$ de risco de morte por doenças cardiovasculares. Aqueles que acumulam entre 18 e 38 min/dia de ATF-MV e que também substituíram o TS por ATF leve diminuíram em $6 \%$ o risco de morte por câncer e em $9 \%$ o risco de morte por doenças cardiovasculares (REES-PUNIA et al., 2019).

Tomadas em conjunto, essas evidências suportam a implementação de estratégias de mudança de comportamento de bombeiros visando o aumento da ATF e a redução do TS, especialmente nos dias de folga. A título de exemplo, duas intervenções em quarteis norte americanos usando estratégias 
de motivação e mudança de comportamento resultaram em impacto positivo no comportamento nutricional, na diminuição do ganho de massa corporal e no bem-estar de bombeiros (ELLIOT et al., 2007). Este tipo de intervenção além de resultar em benefícios individuais podem resultar em economia financeira para a corporação. Após a implementação de programa de promoção da saúde em quarteis norte americanos, foram observadas menores taxas de lesão e menor gasto com atendimento médico entre os participantes (KUEHL et al., 2013). Assim, algumas práticas poderiam ser incluídas em programa de promoção de saúde para bombeiros: 1) estimular a prática regular de ATF e promover escolhas alimentares saudáveis; 2) definir metas e monitorar seu cumprimento; 3) trabalho em equipe multidisciplinar; 4) conhecimento e respeito às individualidade dos participantes; 5) acompanhamento sistemático dos participantes (THE IMAGE STUDY GROUP et al., 2011). 


\section{2 - A Importância da Aptidão Cardiovascular e sua Avaliação no Bombeiro Militar}

Apontamentos históricos indicam que o Corpo Provisório de Bombeiros da Corte, criado em 1856 por D. Pedro II, já se exigia boa qualidade física dos Bombeiros, ainda que sem as evidências científicas hoje disponíveis (ASTE, 1991).

O sucesso e segurança nas operações de bombeiros está relacionado, entre outros fatores, à capacidade física. A alta aptidão cardiorrespiratória (ACR) tem demonstrado ser um dos mais fortes preditores de sobrevida, bem como está associado ao menor risco de doenças crônicas não transmissíveis (HASKELL et al., 2007; KUEHL et al., 2012). Em bombeiros, a necessidade da ACR é observada pela elevada demanda fisiológica da execução de tarefas, com destaque para o combate ao incêndio, como detalhado no tópico 5 . (SMITH et al., 2016).

Tendo esses pressupostos em mente, indaga-se: qual o nível mínimo de ACR que o bombeiro deve possuir para desempenhar suas atividades com segurança? Qual seria o método ideal para avaliarmos a ACR de bombeiros no Brasil, considerando limitações de recursos humanos e financeiros e a logística para se avaliar grandes grupos de militares? Como estimular a manutenção de boa ACR ao longo da carreira?

\section{Qual a ACR recomendada para bombeiros?}

Estudos que simulam tarefas operacionais sugerem que a capacidade mínima aeróbica com o uso de EPI e demandas térmicas, medida pelo consumo de oxigênio $\left(\mathrm{VO}_{2}\right)$, necessária para executar com segurança $\mathrm{O}$ combate a incêndios gira em torno de 33,9 - 45,0 mL(kg.min) ${ }^{-1}$ ou 9,7 - 12,9 METs. MET é a sigla para taxa de equivalente metabólico, sendo que o consumo de energia de um adulto de composição corporal mediana, em repouso, equivale $a \cong 1$ MET. Portanto, uma capacidade máxima de $12 \mathrm{METs}$ 
significa uma tolerância a um esforço máximo correspondente a 12 vezes o repouso. Em um outro estudo, 36 bombeiros usando o EPI realizaram uma tarefa simulada, devendo subir 5 andares, resgatar uma vítima desacordada e descer de volta. A tarefa levou em média 5 min27seg, e o $\mathrm{VO}_{2}$ médio foi de 34,1 $\mathrm{mL}(\mathrm{kg} \cdot \mathrm{min})^{-1}$ ou, 9,7 METs (WILLIAMS-BELL et al., 2010). Em linhas gerais, quanto maior $\mathrm{V}_{2}$ máximo, menor será a sobrecarga relativa causada pela execução de uma tarefa.

Em relação a saúde dos bombeiros, a ACR tem demonstrado ser inversamente relacionada com a prevalência da síndrome metabólica (SM). A prevalência de SM foi quase 10 vezes maior entre os bombeiros com menor ACR ( $\leq 10$ METs) em comparação com aqueles com alta ACR (> 14 METs) (BAUR et al., 2012b).

A NFPA sugere a ACR de 12 METs (cerca de $42 \mathrm{~mL}(\mathrm{~kg} \cdot \mathrm{min})^{-1}$ de $\mathrm{VO}_{2}$ ) como a aptidão física mínima para o desempenho eficiente nas atividades de bombeiros, o que corresponde a uma corrida de $2400 \mathrm{~m}$ no teste de 12 minutos (NATIONAL FIRE PROTECTION ASSOCIATION, 2020). Uma pesquisa apontou que apenas $38,7 \%$ dos bombeiros americanos avaliados atingiam este valor, tendo como VO2 médio o valor de $33,5 \mathrm{~mL}(\mathrm{~kg} \cdot \mathrm{min})^{-1}$. Por outro lado, estudo realizado na Universidade de Brasília - Faculdade de Educação Física, demonstrou ACR mediana de bombeiros brasileiros de 42,4 (18,2 - 64,7) $\mathrm{mL}(\mathrm{kg} \cdot \mathrm{min})^{-1}$, sendo que 52,9\% tiveram $\mathrm{VO}_{2} \max >12 \mathrm{METs}$, valores bem superiores aos apresentados pelos bombeiros americanos (NOGUEIRA et al., 2016).

\section{Medida da Aptidão Cardiorrespiratória nas corporações}

A medida mais precisa para avaliar a ACR é o teste cardiopulmonar, que envolve a realização de esforço em um ergômetro (usualmente uma esteira) e um complexo sistema de análise de gases respirados durante o esforço. Esse tipo de teste é caro, demanda tempo e pessoal altamente qualificado (THOMPSON et al., 2018). Esta sistemática não seria, portanto, factível no 
contexto dos Testes de Aptidão Física (TAF) anuais, em grandes grupos, demandando-se, assim, testes alternativos.

Um dos testes indiretos mais utilizados é o teste de Cooper (teste de 12 min), que é realizado em uma pista de atletismo com 400 metros. Os participantes são orientados a percorrer a máxima distância possível. A equação utilizada leva em consideração a distância máxima percorrida (DP) para estimativa do VO2máx $\left(\mathrm{ml}^{\mathrm{kgg}}{ }^{-1} \cdot \mathrm{min}^{-1}\right)\left[\mathrm{VO}_{2}\right.$ máx $\left.=(\mathrm{DP}-504,1) / 44,79\right] . \mathrm{A}$ avaliação do teste proporciona uma alternativa bastante atraente, uma vez que permite a redução dos custos operacionais e favorece a aplicação em larga escala nas unidades militares e ambientes diversos.

3. Como garantir adequada ACR em bombeiros ao longo da carreira?

No Brasil, a avaliação da ACR é uma das exigências para o ingresso na carreira. Todavia, o uso tradicional de testes físicos antes do ingresso em todas as Forças Armadas do Brasil e em concursos públicos de profissões com reconhecidas demandas físicas, como bombeiros e policiais, fornece uma evidência empírica da viabilidade do seu uso. No entanto, a manutenção de adequada ACR ao longo da carreira ainda é um desafio.

Uma alternativa parece ser a realização regular de TAFs como forma de avaliação periódica e de estímulo, respeitando-se a triagem médicaocupacional prévia como critério de segurança. Desde o ano de 2006 o TAF no CBMDF é realizado anualmente com todos os militares no mesmo local e com os mesmos critérios de avaliação. A partir de um projeto piloto bem-sucedido, a sistemática do TAF se tornou norma institucional. No ano de 2009 foi sancionada a Lei Federal número 12.086, definido, entre outras questões, que o militar deverá obter aproveitamento mínimo de $70 \%$ no TAF, como condição básica para habilitação à promoção a posto superior.

Atualmente, no Corpo de Bombeiros Militar do DF já foram realizadas aproximadamente 50 mil avaliações, onde constam: massa corporal, estatura, pressão arterial, corrida de 12 minutos (teste de Cooper), flexão em barra, flexão abdominal e flexão de braços. O TAF é precedido de triagem médica- 
ocupacional, contribuindo assim para o monitoramento seriado da saúde e aptidão física dos bombeiros. A preocupação com a saúde ao longo da carreira se mostra crítica. Análise desenvolvida no Curso de Altos Estudos para Oficiais do CBMDF, constatou-se que os bombeiros da localidade tiveram uma expectativa de sobrevida média de 5,9 anos a menos do que a população masculina da mesma região (SANTOS, 2011).

\section{A implementação de Políticas Públicas de saúde no CBMDF}

Dentre diferentes políticas de saúde implementadas no CBMDF, destaca-se a inauguração em 2011 do Centro de Capacitação Física e implementação sequencial de academias de ginástica-musculação em suas unidades operacionais. Tal investimento propiciou a otimização da realização dos TAFs em local único e por equipe especializada, além de ser um estímulo à manutenção da aptidão física por aumentar os espaços de prática, podendo gerar benefícios à saúde e qualidade de vida dos bombeiros, além de potencial economia nos gastos em saúde. Entendemos que a disponibilização de local para a prática regular de treinamento físico em todas unidades operacionais é de grande importância para a promoção da saúde e do desempenho profissionais dos bombeiros militares.

\section{3 - A importância do treinamento muscular para bombeiros militares}

A força muscular é um importante componente da aptidão física relacionada à saúde e a sua manutenção em níveis adequados é fundamental para bombeiros (YANG et al., 2019). Nesse sentido, a compreensão sobre os diferentes determinantes da força muscular e o entendimento de como essa variável se conjectura com diferentes marcadores de saúde e de desempenho laboral nessa população é indispensável para o delineamento de políticas institucionais, bem como para a educação dos militares quanto à necessidade do seu envolvimento em programas de treinamento resistido.

Nesse cenário, a idade, o nível de atividade física, a composição corporal, a condição clínica e o hábito de fumar têm sido apontados como 
Artigo Publicado no Vol.06 N.16 - Edição Especial 2020 - ISSN 2359-4829

Versão on-line disponível em: http://www.revistaflammae.com

determinantes da força muscular. Sabe-se que o envelhecimento é acompanhado de um declínio progressivo da força muscular (TIELAND; TROUWBORST; CLARK, 2018), o que é agravado pelo baixo nível de ATF, hábito de fumar e desfechos clínicos negativos como doença coronariana, diabetes, bronquite, hipertensão e asma (STENHOLM et al., 2012). Essas são questões importantes, visto que uma alta prevalência de fatores de risco cardiovascular modificáveis, como sobrepeso e baixos níveis de ATF (DAMACENA et al., 2020), e um risco aumentado de distúrbios respiratórios (RIBEIRO et al., 2009) têm sido identificados em bombeiros militares brasileiros.

Considerando as demandas ocupacionais do bombeiro militar, foi previamente descrita uma correlação negativa entre o tempo despendido em testes que simulam tarefas laborais e os níveis de força de membros superiores $(r=-0,66)$ e força de preensão manual $(r=-0,71)$ (RHEA; ALVAR; GRAY, 2004). Esses achados revelam a importância da força muscular para o desempenho laboral e indicam que sua avaliação pode se dar de modo prático e de baixo custo, por meio da dinamometria manual. Do ponto de vista clínico, foi recentemente demonstrado que um baixo desempenho neuromuscular de membros superiores, medido pelo teste de flexão de braços, se associou à maior incidência de doenças cardiovasculares em bombeiros (YANG et al., 2019).

Diante da relevância da força muscular para a saúde e o desempenho funcional de bombeiros militares, sugere-se a inclusão do treinamento de força em programas de exercícios físicos para essa população. Além do aumento da força propriamente dita, a prática de exercícios resistidos está associada à redução do risco de doenças cardiometabólicas e câncer (MCLEOD; STOKES; PHILLIPS, 2019). Adicionalmente, o treinamento resistido pode contribuir para o tratamento de distúrbios musculoesqueléticos como dor lombar e lesões no joelho, que são os locais mais acometidos em bombeiros (PHELPS et al., 2018). Estudos apontam ainda benefícios do treinamento resistido na saúde mental. Um estudo de revisão envolvendo 1877 participantes demonstrou que 
a prática de exercícios resistidos pode reduzir os sintomas de depressão independente do status de saúde, volume de treinamento ou dos ganhos de força (GORDON et al., 2018).

Devido a carência de recomendações específicas para bombeiros militares, a prescrição do treinamento resistido para esses profissionais pode ser feita a partir das recomendações que envolvem a população geral, como as descritas pelo American College of Sports Medicine (GARBER et al., 2011). No entanto, é fundamental que estímulos que se aproximem das tarefas físicas profissionais sejam inseridos no programa de treinamento, bem como os exercícios envolvidos nos TAFs. Deve se considerar também o envolvimento do profissional em outros programas de treinamento físico/atividade física dentro ou fora do cenário institucional, que, somando-se à sobrecarga da profissão, podem resultar em estímulos em demasia, podendo gerar consequências negativas do excesso de treinamento (overreaching e overtraining), o que aumenta o risco de lesões.

Em resumo, a prática do treinamento resistido pode aprimorar diversos marcadores de saúde, melhorar o desempenho laboral, contribuir para o tratamento ou prevenção de distúrbios musculoesqueléticos, decorrentes ou não da prática profissional, e também contribuir para a melhora da saúde mental de bombeiros militares. Em tempo, essas adaptações podem também contribuir para a redução do número de afastamentos por motivos de doença e melhorar a qualidade dos serviços prestados à população.

\section{4 - Composição corporal e a aptidão física}

Entre os componentes da aptidão física, a composição corporal se destaca entre os demais estudos, principalmente pela sua ligação com a saúde. O termo composição corporal refere pela necessidade de entender melhor os componentes da massa corporal, visto que tanto o excesso de gordura quanto a baixa massa muscular podem significar prejuízo para a saúde e o desempenho ocupacional. 
A Organização Mundial da Saúde (OMS) recomenda o uso do índice de massa corporal (IMC) como um instrumento de avaliação pelo seu fácil uso, embora reconheça suas limitações. Segundo as diretrizes da OMS, IMC $\geq 25$ $\mathrm{kg} / \mathrm{m}^{2}$ corresponde à categoria de excesso de peso e $I M C \geq 30 \mathrm{~kg} / \mathrm{m}^{2}$ à obesidade, que é um importante fator de risco para doenças cardiovasculares, diabetes, problemas musculoesqueléticos, câncer e apneia do sono, entre outros (SOTERIADES et al., 2011; WORLD HEALTH ORGANIZATION, 2020). Além disso, estudos mostram associação com impactos negativos da obesidade na saúde mental e na qualidade de vida (ALBANO et al., 2019; LUPPINO et al., 2010).

Em bombeiros, essa condição também merece atenção. Estudos apontam um risco 7 vezes maior de bombeiros com obesidade apresentarem hipertensão arterial quando comparados com bombeiros não obesos (SOTERIADES et al., 2011). Ainda, a chance de morte súbita de bombeiros em serviço, causada por doença coronariana, foi aumentada entre 1,5 e 6,6 vezes em comparação com bombeiros não obesos (KALES et al., 2003). Obesidade é um fator de risco para a incidência de doenças coronarianas, incapacidade relacionada ao trabalho, absenteísmo e aposentadoria precoce em bombeiros (POSTON et al., 2011; SOTERIADES et al., 2011).

Devido a elevada exigência ocupacional, espera-se que bombeiros apresentem maior massa muscular que a população em geral, o que poderia superestimar a obesidade em bombeiros a partir do IMC. Entretanto, estudos em bombeiros americanos e brasileiros mostram que não. O IMC foi um indicador confiável de obesidade entre bombeiros. Porto et al. (2016) avaliaram em 4216 bombeiros militares a precisão do IMC em detectar obesidade comparativamente ao uso do percentual de gordura corporal. Ao contrário do esperado, a prevalência de obesidade pelo IMC $(13,3 \%)$ foi menor do que usando o percentual de gordura corporal (15,9\%). Diferentemente da obesidade, a avaliação da categoria de excesso de peso (IMC entre 25,0 $29,9 \mathrm{~kg} / \mathrm{m}^{2}$ ) está mais sujeita a erros, indicando cautela no uso do IMC para esta finalidade (JITNARIN et al., 2013). 
Outro ponto que merece destaque é a relação da obesidade com a aptidão física em bombeiros. Um estudo realizado com 3.822 bombeiros militares brasileiros demonstrou associação entre menor ACR e resistência muscular localizada (número de abdominais) com maiores valores de IMC (PORTO et al., 2016). Semelhantemente, um estudo com 4.237 bombeiros investigou a associação entre obesidade e ACR. Bombeiros com IMC $\geq 30 \mathrm{~kg} / \mathrm{m}^{2}$ possuíam uma chance 6 a 9 vezes maior de estarem com uma ACR inadequada para a atividade profissional em comparação com bombeiros com IMC $<30 \mathrm{~kg} / \mathrm{m}^{2}$ (NOGUEIRA et al., 2016). Outro estudo com bombeiros do Espírito Santo observou que ter obesidade central (cintura $>94 \mathrm{~cm}$ ) aumentava a chance de ter ACR inadequada em 3 a 8 vezes mais comparado a quem tinha cintura <94 cm (DAMACENA et al., 2020).

Ressalta-se ainda a importância da atividade física para manutenção da ACR. Baur et al (2012a) observaram que apesar de bombeiros com obesidade possuírem ACR menor que seus pares sem obesidade, ela era maior entre bombeiros obesos que faziam 150 minutos ou mais de atividade física por semana em relação aqueles que não o faziam. É importante realçar que a obesidade é uma condição multifatorial e complexa (WORLD HEALTH ORGANIZATION, 2020). Assim, a estratégia deve ser sempre de acolher e dar suporte aos indivíduos quando se objetiva prevenir ou tratar o excesso de peso (PUHL; HEUER, 2010).

Korre e colaboradores (2017) realizaram uma revisão sobre intervenções dietéticas em bombeiros. Alguns pontos importantes para intervenções bemsucedidas são a educação e o acesso fácil a informação de qualidade sobre nutrição e qualidade na alimentação. Bombeiros costumam realizar muitas refeições aquartelados, assim, é importante que a mudança de hábitos alimentares ocorra também no quartel, envolvendo o preparo de alimentos e a redução do consumo de refrigerantes e fast-food. A principal proposta de Korre et al. (2017) é o uso da dieta mediterrânea devido a pesquisas que mostram reduções significativas da incidência de cânceres na população em geral, bem como de doenças cardiovasculares. Seus dados preliminares apontam que 
bombeiros com maior aderência à dieta mediterrânea possuíam chance 35\% menor de terem síndrome metabólica e $43 \%$ de ganho de peso nos últimos 5 anos.

\section{5 - Riscos à saúde associados à atividade de bombeiro - uma visão global}

A abordagem de riscos à saúde relacionados à profissão de bombeiro é ampla e complexa. Os riscos ocupacionais se associam ao tipo de atividade desenvolvida, como busca e salvamento, combate a incêndios e/ou o atendimento a emergências médicas, às características gerais da profissão, como o trabalho em regime de escala, a execução de jornadas de trabalho extraordinárias, o baixo poder decisório, os maus hábitos alimentares associados à disponibilidade nas proximidades dos locais de trabalho e às condições clínico-funcionais individuais, como o grau de aptidão física, a composição corporal ou a saúde cardiovascular (SOTERIADES et al., 2011). Esses profissionais estão sujeitos aos riscos ambientais que afetam a população em geral, acrescidos daqueles inerentes à profissão. Destacam-se os riscos cardiovasculares, metabólicos e de desenvolvimento de tumores malignos e de distúrbios psicológicos (GAINEY et al., 2018; KALES; SMITH, 2017; SOTERIADES et al., 2019).

$\mathrm{Na}$ apreciação do risco, destacaremos duas questões: a tarefa e as características individuais. Existem tanto determinantes ambientais / ocupacionais para o maior ou o menor risco, quanto determinantes individuais. Assim, não é qualquer atividade que tem risco ocupacional elevado, assim como o risco não é igual entre todos os bombeiros, mesmo que nas mesmas atividades. A questão fundamental é, portanto, identificar características sociodemográficas, ambientais, profissionais e/ou individuais que aumentem a susceptibilidade para agravos à saúde do bombeiro. O passo seguinte é traçar estratégias ou políticas públicas nas corporações para a identificação dos indivíduos mais suscetíveis e agir preventivamente. 
No estudo que identificou que cerca de $45 \%$ dos casos de morte súbita em serviço de bombeiros americanos foram causadas por doenças cardíacas, observou-se também uma nítida associação entre as causas de morte e a tarefa desenvolvida. $32,1 \%$ das mortes ocorreram durante o combate a incêndios, $13,4 \%$ na fase de atendimento ao alarme, 17,4\% no retorno após atendimento, $12,5 \%$ em treinamento físico, 9,5\% em atendimentos a emergências sem fogo e 15,4\% em situações de não-emergência. O maior número de mortes $(32,1 \%)$ ocorreu na atividade em que os bombeiros passavam o menor tempo de suas jornadas anuais, o combate a incêndio, com cerca de 1 a $5 \%$ do tempo de trabalho. O risco de morte por doença arterial coronariana foi 136 vezes maior no combate a incêndio, que aquele enfrentado em atividades de não-emergência (KALES et al., 2007), indicando assim a importância de se considerar a tarefa realizada quando da análise de risco.

Por outro lado, um projeto que avaliou casos de morte súbita em serviço de bombeiros americanos identificou que aqueles que apresentaram, no exame de autópsia, doença arterial coronariana (algum grau de obstrução nas artérias que irrigam o coração), infarto do miocárdio ou coração aumentado (cardiomegalia ou hipertrofia ventricular esquerda) tiveram risco de morte súbita cardíaca de 9,3; 6,2 e 6,1 vezes a mais, respectivamente, comparados com aqueles que também morreram em serviço, mas de causas não cardíacas (SMITH et al., 2018). Este estudo gerou evidências que sugerem o uso de exames específicos na rotina de triagem médico-ocupacional de bombeiros visando a identificação precoce dessas condições clínicas, como estratégia preventiva para a redução dos casos de morte súbita cardíaca em serviço (SMITH et al., 2018). Percebe-se então como as condições individuais também devem ser consideradas nas análises de risco.

Neste mesmo projeto se estudou a associação da tarefa com algumas condições clínicas individuais, combinando assim questões ocupacionais e pessoais. Foram avaliadas somente as fatalidades em serviço de origem cardíaca. 80\% desses bombeiros apresentavam evidências de doença arterial coronariana e aumento do coração, corroborando os achados do estudo 
anterior (SMITH et al., 2018) e reforçando a sugestão de triagem médicoocupacional para essas condições. Outro achado de destaque foi a constatação de que os bombeiros deste grupo que estavam em atividades de combate a incêndio apresentaram risco de morte súbita cardíaca 112 vezes maior que aqueles que desempenhavam atividades no quartel de nãoemergência (SMITH et al., 2019). Esses dados, à semelhança de achados anteriores (KALES et al., 2007), demonstram o maior risco cardiovascular durante $O$ combate a incêndio, comparativamente à outras tarefas ocupacionais.

Importante destacar que não dispomos de séries históricas de mortalidade em serviço de bombeiros brasileiros. Entretanto as sobrecargas são bastante semelhantes e dados de menor expectativa de vida dos bombeiros brasileiros, comparativamente à população em geral, reforçam os riscos da profissão (SANTOS, 2011). Ademais, e a despeito de muitos exemplos serem baseados em dados de bombeiros americanos, eles foram usados neste tópico para demonstrar que os riscos se associam tanto às tarefas e rotinas profissionais, quanto às condições clínico funcionais individuais. Essa lógica, em princípio, se aplica a qualquer corporação.

Os achados indicam a necessidade de triagem médico-ocupacional para identificação precoce de indivíduos mais suscetíveis a maiores riscos, assim como pela implementação de estratégias de atribuição de tarefas segundo as condições clínicas e funcionais de cada um. Este é um cenário complexo, especialmente em situações com recursos humanos e financeiros escassos, o que demanda esforço conjunto daqueles que atuam prioritariamente na pesquisa (universidades, institutos, etc.) com a corporações de bombeiros, detentoras da expertise prática.

Quanto à prevenção ou à redução do risco, é fundamental considerar o potencial fator protetor da prática de atividade física e da boa ACR (NOGUEIRA, 2016; DURAND et al., 2011). É importante também considerar que os riscos, tanto profissionais quanto individuais, são multifatoriais. Em um artigo ilustrativo deste cenário, dois dos maiores pesquisadores na área 
apresentam uma figura esquemática mostrando que os riscos devem considerar, entre outros, 1 - o tipo de tarefa desempenhada; 2 - as condições agudas (momentâneas) do profissional, como o grau de repouso prévio e seu nível de hidratação e 3 - as condições individuais quanto, por exemplo, a idade, o estilo de vida, a composição corporal, o nível de aptidão física e a presença de doença prévia (KALES; SMITH, 2017). Assim, menor idade, adequada composição corporal, boa aptidão física, ausência de doenças e tarefas ocupacionais de menor exigência apontam para menores riscos. Por outro lado, idade mais avançada, falta de repouso adequado antes do plantão, desidratação, obesidade, baixa ACR, existência de doença prévia e a realização de tarefas mais exigentes, especialmente o combate a incêndio, apontando para maiores riscos.

Nesta visão global de riscos, é importante mencionar uma condição específica e bastante frequente e em nossa região: o incêndio florestal. Em um estudo de mestrado identificamos elevada sobrecarga cardiovascular e grande acúmulo de atividade física em serviço desses profissionais (SAINT MARTIN, 2018). Existem também evidências que apontam a piora em indicadores cardiometabólicos, como perfil lipídico e gordura corporal, após cerca de 3 meses de trabalho de bombeiros americanos na estação de incêndios florestais (COKER et al., 2019). Os identificaram que o potencial benefício metabólico do maior gasto energético durante a ação pode ser suplantado pelos efeitos negativos da oferta inadequada de alimentos saudáveis nos locais de combate ao incêndio, do estresse, de alterações do sono e da prolongada exposição à fumaça (COKER et al., 2019).

Vale mencionar ainda que as rotinas / escalas de trabalho também poder exercer um papel na complexa análise dos riscos. O gerenciamento de escalas é sabidamente complexo, seja pela cultura organizacional ou pela própria demanda. Entretanto, há que se considerar evidências mostrando a relação entre o aumento no número de plantões no mês com a hipertensão arterial e a obesidade (CHOI et al., 2016; CHOI; SCHNALL; DOBSON, 2016). 
Faz-se necessário ainda considerar algumas especificidades de gênero. As mulheres ingressaram mais recentemente nos corpos de bombeiro. No Distrito Federal o ingresso se deu em 1993 (CBMDF, 2020). Seja pelo menor tempo de participação na carreira ou pelo menor contingente, as mulheres têm sido historicamente menos avaliadas no âmbito da pesquisa. Felizmente, novos estudos têm se dedicado a estudar também as mulheres em suas especificidades na profissão. Um levantamento entre 840 bombeiras de 14 países observou elevados percentuais de respondentes que consideravam que o ciclo menstrual e a menopausa afetam suas capacidades de trabalho. Parece ainda haver reduzida oferta de equipamentos de proteção individual específicos para mulheres. Outra importante constatação foi que metade das participantes alegavam ter pouco apoio para treinamento físico (WATKINS et al., 2019). A questão do treinamento físico e sua correspondente exigência de critérios mínimos é alvo de amplo debate, tanto na pesquisa quanto nas corporações. Existe razoável consenso no campo da fisiologia do exercício de que o desempenho aeróbico médio dos homens é maior que o das mulheres, dentro da mesma faixa etária, ressalvado o fato de que as diferenças vêm diminuindo nas últimas décadas, provavelmente em função de fatores socioculturais, como a maior participação das mulheres em atividades esportivas. Entre 57.284 pessoas (sendo $51,5 \%$ de homens) observou que a ACR das mulheres foi cerca de 2 METs a menos que a dos homens (AL-MALLAH et al., 2016). Este fenômeno subsidia o estabelecimento de menores parâmetros mínimos de exigência de aptidão física para as mulheres. Por outro lado, há quem defenda que as exigências sejam semelhantes, contrariando de certa forma as evidências fisiológicas, sob o argumento de que as exigências do trabalho são as mesmas, seja para homens, mulheres, pessoas mais novas, mais velhas, etc. Essa discussão ultrapassa os objetivos deste artigo, mas é importante considerar que outras questões além da capacidade física devem ser consideradas. Em bombeiras da província do Quebec, no Canadá, observou-se elevada proporção de participantes com risco cardiovascular moderado ou alto, com destaque para $12 \%$ de obesidade e $62 \%$ de atividade física insuficiente. 
Em nosso grupo, avaliamos a qualidade de vida e outros parâmetros entre 687 homens e 104 mulheres militares do CBMDF. A qualidade de vida foi estatisticamente semelhante entre os grupos e também observamos maior ACR média entre os homens. Entretanto, o percentual de participantes fisicamente ativos foi maior entre as mulheres (SEGEDI, 2018). O cenário indica fortemente pela necessidade de mais estudos nesse grupo.

\section{6 - O papel do sono na saúde global e cardiovascular do bombeiro militar}

A importância do sono para a saúde tem sido cada vez mais estudada e muita atenção tem sido dada para grupos que trabalham por escala, especialmente quando isso inclui o trabalho noturno (NARCISO et al., 2016). Dormir faz parte do processo natural da vida e é uma necessidade fisiológica. Apesar disso, a duração de sono por noite tem sido reduzida com o passar dos anos. Embora a média de sono da população seja entre 7-8h de sono por noite, tem-se observado aumento progressivo da prevalência de indivíduos que dormem menos de 6h (FORD; CUNNINGHAM; CROFT, 2015). O sono pode ser avaliado sob duas perspectivas, uma objetiva (duração, latência, distúrbios relacionados ao sono) e outra subjetiva (relacionadas à percepção da qualidade do sono do indivíduo). Uma pesquisa de revisão demonstrou que aqueles com o sono curto $(<6 \mathrm{~h})$, comparados com aqueles que apresentaram sono normal, tinham um aumento significativo na mortalidade por todas as causas, diabetes, doenças cardiovasculares e obesidade (ITANI et al., 2017).

No contexto da saúde ocupacional, o relatório de fatalidades de bombeiros da NFPA nos Estados Unidos listou as principais causas de morte de bombeiros em serviço, sendo a primeira as de origem cardiovascular, como já abordado, e a segunda acidentes com veículos motorizados (FAHY; MOLIS, 2009). Tendo em vista a grande relação das principais causas de morte com o sono, um estudo investigou através de questionários a associação entre distúrbios do sono e o risco de acidentes de veículos automotor em 6.933 bombeiros. $37,2 \%$ dos participantes apresentaram pelo menos um distúrbio do sono e 2 vezes mais chances de sofrer um acidente de automóvel comparados 
com quem não apresentou nenhum distúrbio do sono. Ainda, os bombeiros sob risco de distúrbios do sono tiveram risco aumentando em mais de 2 vezes para doença cardiovascular ou diabetes e em mais de 3 vezes para depressão ou ansiedade (BARGER et al., 2015).

Um programa de intervenção para melhoria do sono conduzido entre bombeiros americanos com sessões educacionais obrigatórias sobre higiene do sono, triagem voluntária de distúrbios do sono, diagnóstico e tratamento demonstrou que os bombeiros designados para os quarteis onde a intervenção foi realizada relataram em média $46 \%$ menos dias de incapacidade por lesão do que aqueles designados para os quarteis controle (sem intervenção). A intervenção também se mostrou eficiente para reduzir as lesões relatadas em $10 \%$ ao ano, o percentual de bombeiros feridos em 17\%, resultando em maior efetivo de trabalho à disposição do departamento (SULLIVAN et al., 2017).

Desta forma, ao planejar políticas de intervenções nas corporações, podem ser utilizados instrumentos de avaliação direta e indireta que avaliam o sono de modo objetivo e subjetivo. Dentre os instrumentos mais comuns de avaliação do sono, existe o Índice de Qualidade de Sono de Pittsburgh (IQSP) que é ferramenta validada para o português, que pode ser aplicado em larga escala e de baixo custo, com potencial tanto para a pesquisa quanto em avaliações periódicas de saúde ocupacional (BERTOLAZI et al., 2011).

\section{7 - Função autonômica cardíaca e frequência cardíaca como indicadores de saúde cardiovascular}

A saúde cardiovascular é uma condição imperiosa para a realização com segurança e eficácia dos múltiplos tipos de emergências no qual normalmente o bombeiro está envolvido. Apesar de outros riscos ocupacionais, como de queimaduras, inalação de fumaça e colapso de estruturas, a maior proporção de fatalidades em serviço está ligada a eventos cardíacos, como comentado no tópico 5.

Bombeiros estão envolvidos na realização de complexas e demandantes tarefas que geram elevado gasto energético, exigindo do aparelho 
cardiovascular pronto fornecimento de suficiente, contínuo e variável fluxo sanguíneo aos diversos tecidos e órgãos frente à maior demanda de oxigênio e nutrientes, visando 0 atendimento das exigências funcionais orgânicas desencadeadas (JUNQUEIRA, 2015). Nesse cenário dos ajustes cardiovasculares frente às demandas impostas ao bombeiro, destacam-se diversos mecanismos reguladores cardiovasculares para a manutenção das funções orgânicas. Abordaremos somente o mecanismo de atuação neural. 0 coração está sob a influência reguladora do sistema nervoso autônomo, em suas divisões simpática (estimuladora sobre o coração) e a parassimpática (inibitória sobre coração)(JUNQUEIRA, 2015). A ação contínua e reflexa dos ramos simpático e parassimpático sob o coração resulta na frequência cardíaca (FC) observada nas diferentes demandas funcionais, ou seja, a FC é a resultante do balanço das atividades dos ramos simpático e parassimpático sob o coração. Essa dinâmica integrativa é chamada de função autonômica cardíaca (FAC).

A avaliação da FAC pode ser obtida por diferentes métodos, dentre os quais destacam-se dois: (a) a variabilidade da frequência cardíaca (VFC) e (b) a frequência cardíaca de recuperação (FCR) (LAHIRI; KANNANKERIL; GOLDBERGER, 2008). A VFC é um método baseado no registro curto ou longo das oscilações dos intervalos entre os batimentos cardíacos sendo um método adequado para acessar o balanço simpático e parassimpático no coração (MALIK et al., 2019).

Com relação a FCR sabe-se que durante o esforço físico a FC aumenta em resposta simultânea da ativação simpática e desativação parassimpática e que após o esforço, nos momentos iniciais de recuperação, ocorre a adaptação em coativação da progressiva desativação simpática e rápida reativação parassimpática reduzindo a FC para próximo dos valores de repouso (IMAI et al., 1994).

Vários estudos demostram que ambos os métodos de avaliação da FAC são considerados marcadores de morbimortalidade e de saúde na população em geral (DEKKER et al., 1997; QIU et al., 2017). No estudo que acompanhou 
885 homens por 25 anos, foi verificado que a taxa relativa de mortalidade foi $110 \%$ maior para indivíduos com baixa VFC comparativamente àqueles com maior VFC (QIU et al., 2017). Com relação a FCR um estudo de revisão com 34.267 participantes em que os autores compararam a lenta FCR com rápida FCR, verificou-se que a lenta FCR apresentou risco aumentado de $69 \%$ para eventos cardiovasculares e $68 \%$ para mortalidade por todas as causas. Para cada 10bpm de diminuição na recuperação da FC (recuperação mais lenta), o risco aumentou em 13\% para eventos cardiovasculares e em 9\% para mortalidade geral (DEKKER et al., 1997). Portanto do ponto de vista fisiológico, a baixa VFC no repouso e/ou prejuízo na recuperação da FCR estão diretamente associados com a redução da capacidade de modulação da FAC. Assim, os autores recomendam fortemente a realização do registro da VFC e da FCR como rotina para avaliação de risco na prática clínica (DEKKER et al., 1997; QIU et al., 2017).

Como mencionado, a NFPA (1852)propõe um mínimo de 12 METs de ACR para bombeiros. Sabe-se que a aptidão cardiorrespiratória se associa positivamente grau de modulação da FAC (YAMAMOTO et al., 2001). O estudo realizado por nosso grupo que comparou a FAC em bombeiros no serviço (quartel) e fora do serviço (laboratório) verificou predominância simpática em ambas as condições. Entretanto, após utilizar os 12 METs de ACR como referência, observamos associação positiva entre a CRF e a atividade parassimpática (PORTO et al., 2019a).

Em estudo que avaliou os efeitos da ACR e de 10 semanas de treinamento físico na dinâmica da FC durante e após simulado de tarefas de combate ao fogo, os resultados demonstraram que o treinamento físico foi capaz de aumentar o decremento da FCR nos 60 segundos iniciais de recuperação. Os autores concluíram que o treinamento físico está associado ao menor estresse fisiológico e recuperação acelerada nas tarefas de simulação de combate ao incêndio (SAARI, 2019).

Em geral, as pesquisas apontam para dois aspectos fundamentais com relação a FAC que devem ser observados em bombeiros. Os achados indicam 
para o benefício de: a) a avaliação seriada da FAC, por meio de métodos como a VFC e a FCR, por exemplo e b) o exercício físico regular com foco na capacidade cardiopulmonar visando o potencial efeito cardioprotetor.

\section{Considerações finais}

Diante da complexidade da demanda profissional e dos riscos a que bombeiros militares são expostos em suas rotinas diárias, algumas práticas bem documentadas na literatura especializada merecem destaque. Dispomos de suficiente evidência científica para a recomendação de: a) treinamento físico sistemático (aeróbico e muscular); b) incentivo à prática regular de atividades físicas; c) incentivo à redução do tempo sentado; d) implementação de estratégias para escolhas alimentares saudáveis nas corporações; e) incremento contínuo das rotinas de triagem médico-ocupacional e f) políticas com foco na melhoria da qualidade do sono, como estratégias fundamentais para o aumento da segurança e a redução dos agravos à saúde desses profissionais, visando salvar vidas e promover a qualidade de vida. Esses tópicos não esgotam o assunto, visto que áreas como a saúde mental e os riscos de cânceres associados à profissão, que são tão importantes quanto as demais, não foram aqui discutidas.

Os desafios para se alcançar esses objetivos são grandes e passam, entre outras questões, pela imperiosa necessidade de se otimizar rotinas para a identificação precoce daqueles militares mais suscetíveis a agravos à saúde, tendo também como elemento norteador o tipo de tarefa a ser desempenhada. A implementação contínua de políticas internas, baseadas em evidências científicas e com foco na promoção da saúde e da segurança do bombeiro, é de extrema importância. Neste contexto, ênfase deve ser dada ao contínuo aperfeiçoamento das rotinas de triagem médico-ocupacional, com vistas à identificação precoce de situações e/ou indivíduos mais suscetíveis ao risco.

Neste grande desafio, sugere-se a união contínua de esforços entre a ciência, por meio de parcerias com universidades, institutos de pesquisa e grupos de estudo, e as corporações de bombeiros do Brasil e do mundo, 
Artigo Publicado no Vol.06 N.16 - Edição Especial 2020 - ISSN 2359-4829

Versão on-line disponível em: http://www.revistaflammae.com

detentoras da expertise prática. Aproveitamos esse espaço privilegiado de contato com bombeiros militares de todo o Brasil para, como Grupo de Pesquisa da Faculdade de Educação Física da Universidade de Brasília - FEFUnB, nos colocarmos à disposição para todo apoio que possamos oferecer nesta perspectiva. Nossa parceria com o Corpo de Bombeiros Militar do Distrito Federal (CBMDF) tem sido absolutamente frutífera e fonte de inspiração para nosso trabalho na Universidade de Brasília, buscando contribuir de forma qualificada com a sociedade brasileira.

Enquanto Grupo de Pesquisa com forte ênfase no estudo de indicadores de saúde e qualidade de vida de bombeiros, e dos riscos inerentes à profissão, nosso lema é de que todo esforço será válido se de alguma forma contribuirmos na produção de conhecimento ou na intervenção prática para promover a saúde e o desempenho profissional daqueles e daquelas que estão diuturnamente protegendo a sociedade e salvando vidas. Se nosso esforço for capaz de salvar a vida de um(a) único(a) bombeiro(a), promover a saúde de um(a) único(a) bombeiro(a) ou de melhorar a qualidade de vida de um(a) único(a) bombeiro(a), TUDO TERÁ VALIDO A PENA!

\section{Referências:}

ALBANO, G. et al. Interpersonal difficulties in obesity: A systematic review and meta-analysis to inform a rejection sensitivity-based model. Neuroscience and Biobehavioral Reviews, v. 107, p. 846-861, 2019.

AL-MALLAH, M. H. et al. Sex Differences in Cardiorespiratory Fitness and AllCause Mortality: The Henry Ford Exerclse Testing (FIT) Project. Mayo Clinic Proceedings, v. 91, n. 6, p. 755-762, 2016.

ASTE, G. História do Corpo de Bombeiros. Rio de Janeiro. Imprensa Oficial do Estado do Rio de Janeiro.1991.

BARGER, L. K. et al. Common Sleep Disorders Increase Risk of Motor Vehicle Crashes and Adverse Health Outcomes in Firefighters. Journal of Clinical Sleep Medicine : JCSM : Official Publication of the American Academy of Sleep Medicine, v. 11, n. 3, p. 233-240, 15 mar. 2015. 
BAUR, D. M. et al. Age-Related Decline in Cardiorespiratory Fitness among Career Firefighters: Modification by Physical Activity and Adiposity. Journal of Obesity, v. 2012, p. 1-6, 2012a.

BAUR, D. M. et al. Low fitness is associated with exercise abnormalities among asymptomatic firefighters. Occupational Medicine, v. 62, n. 7, p. 566-569, 1 out. 2012b.

BERTOLAZI, A. N. et al. Validation of the Brazilian Portuguese version of the Pittsburgh Sleep Quality Index. Sleep Medicine, v. 12, n. 1, p. 70-75, jan. 2011.

BUMAN, M. P. et al. Reallocating Time to Sleep, Sedentary Behaviors, or Active Behaviors: Associations With Cardiovascular Disease Risk Biomarkers, NHANES 2005-2006. American Journal of Epidemiology, v. 179, n. 3, p. 323-334, 1 fev. 2014.

CBMDF. Corpo de Bombeiros Militar do Distrito Federal. Disponível em: $<$ https://www.cbm.df.gov.br>. Acesso em: 11 jun. 2020.

$\mathrm{CHOI}$, B. et al. 24-hour work shifts, sedentary work, and obesity in male firefighters. American Journal of Industrial Medicine, v. 59, n. 6, p. 486-500, 2016.

CHOI, B.; SCHNALL, P.; DOBSON, M. Twenty-four-hour work shifts, increased job demands, and elevated blood pressure in professional firefighters. International Archives of Occupational and Environmental Health, v. 89, n. 7, p. 1111-1125, out. 2016.

COKER, R. H. et al. Wildland Firefighting: Adverse Influence on Indices of Metabolic and Cardiovascular Health. Journal of Occupational and Environmental Medicine, v. 61, n. 3, p. e91-e94, mar. 2019.

DAMACENA, F. C. et al. Obesity prevalence in Brazilian firefighters and the association of central obesity with personal, occupational and cardiovascular risk factors: a cross-sectional study. BMJ open, v. 10, n. 3, p. e032933, 12 mar. 2020.

DEKKER, J. M. et al. Heart rate variability from short electrocardiographic recordings predicts mortality from all causes in middle-aged and elderly men. The Zutphen Study. American Journal of Epidemiology, v. 145, n. 10, p. 899-908, 15 maio 1997.

DURAND, G. et al. Firefighters' Physical Activity: Relation to Fitness and Cardiovascular Disease Risk. Medicine \& Science in Sports \& Exercise, v. 43, n. 9, p. 1752-1759, set. 2011. 
DUVIVIER, B. M. F. M. et al. Benefits of Substituting Sitting with Standing and Walking in Free-Living Conditions for Cardiometabolic Risk Markers, Cognition and Mood in Overweight Adults. Frontiers in Physiology, v. 8, p. 353, 8 jun. 2017.

EKELUND, U. et al. Does physical activity attenuate, or even eliminate, the detrimental association of sitting time with mortality? A harmonised metaanalysis of data from more than 1 million men and women. The Lancet, v. 388, n. 10051, p. 1302-1310, 2016.

ELLIOT, D. L. et al. The PHLAME (Promoting Healthy Lifestyles: Alternative Models??? Effects) Firefighter Study: Outcomes of Two Models of Behavior Change: Journal of Occupational and Environmental Medicine, v. 49, n. 2, p. 204-213, fev. 2007.

FAHY, R. F.; MOLIS, J. L. Firefighter Fatalities in the US-2018. NFPA, 2009.

FORD, E. S.; CUNNINGHAM, T. J.; CROFT, J. B. Trends in Self-Reported Sleep Duration among US Adults from 1985 to 2012. Sleep, v. 38, n. 5, p. 829832, 1 maio 2015.

GAINEY, S. J. et al. Exposure to a firefighting overhaul environment without respiratory protection increases immune dysregulation and lung disease risk. PLoS ONE, v. 13, n. 8, 21 ago. 2018.

GARBER, C. E. et al. American College of Sports Medicine position stand. Quantity and quality of exercise for developing and maintaining cardiorespiratory, musculoskeletal, and neuromotor fitness in apparently healthy adults: guidance for prescribing exercise. Med Sci Sports Exerc, v. 43, n. 7, p. 1334-59, jul. 2011.

GORDON, B. R. et al. Association of Efficacy of Resistance Exercise Training With Depressive Symptoms: Meta-analysis and Meta-regression Analysis of Randomized Clinical Trials. JAMA psychiatry, v. 75, n. 6, p. 566-576, 01 2018.

GUTHOLD, R. et al. Worldwide trends in insufficient physical activity from 2001 to 2016: a pooled analysis of 358 population-based surveys with 1.9 million participants. The Lancet Global Health, v. 6, n. 10, p. e1077-e1086, out. 2018.

HASKELL, W. L. et al. Physical activity and public health: updated recommendation for adults from the American College of Sports Medicine and the American Heart Association. Circulation, v. 116, n. 9, p. 1081, 2007.

IMAI, K. et al. Vagally mediated heart rate recovery after exercise is accelerated in athletes but blunted in patients with chronic heart failure. Journal of the American College of Cardiology, v. 24, n. 6, p. 1529-1535, 15 nov. 1994. 
Artigo Publicado no Vol.06 N.16 - Edição Especial 2020 - ISSN 2359-4829

Versão on-line disponível em: http://www.revistaflammae.com

ITANI, O. et al. Short sleep duration and health outcomes: a systematic review, meta-analysis, and meta-regression. Sleep Medicine, v. 32, p. 246-256, abr. 2017.

JITNARIN, N. et al. Accuracy of body mass index-defined overweight in fire fighters. Occupational Medicine (Oxford, England), v. 63, n. 3, p. 227-230, abr. 2013.

JUNQUEIRA, J., LUIZ. CONSIDERAÇÕES BÁSICAS SOBRE A ORGANIZAÇÃO ESTRUTURAL E A FISIOLOGIA DO APARELHO CARDIOVASCULAR.

Disponível em:https://www.researchgate.net/publication/279173038_CONSIDERACOES BASICAS SOBRE_A_ORGANIZACAO_ESTRUTURAL_E_A_FISIOLOGIA_D O_APARELHO_CARDIOVASCULAR.. Acesso em: 15 Jun. 2020.

KALES, S. N. et al. Firefighters and on-duty deaths from coronary heart disease: a case control study. Environmental Health: A Global Access Science Source, v. 2, n. 1, p. 14, 6 nov. 2003.

KALES, S. N. et al. Emergency duties and deaths from heart disease among firefighters in the United States. New England Journal of Medicine, v. 356, n. 12, p. 1207-1215, 2007.

KALES, S. N.; SMITH, D. L. Firefighting and the Heart: Implications for Prevention. Circulation, v. 135, n. 14, p. 1296-1299, 4 abr. 2017.

$\mathrm{KOHL}, \mathrm{H}$. W. et al. The pandemic of physical inactivity: global action for public health. The Lancet, v. 380, n. 9838, p. 294-305, 21 jul. 2012.

KORRE, M.; SOTOS-PRIETO, M.; KALES, S. N. Survival Mediterranean Style: Lifestyle Changes to Improve the Health of the US Fire Service. Frontiers in Public Health, v. 5, p. 331, 2017.

KUEHL, K. S. et al. Body Mass Index As a Predictor of Firefighter Injury and Workers' Compensation Claims: Journal of Occupational and Environmental Medicine, v. 54, n. 5, p. 579-582, maio 2012.

KUEHL, K. S. et al. Economic benefit of the PHLAME wellness programme on firefighter injury. Occupational Medicine, v. 63, n. 3, p. 203-209, 1 abr. 2013.

LAHIRI, M. K.; KANNANKERIL, P. J.; GOLDBERGER, J. J. Assessment of autonomic function in cardiovascular disease: physiological basis and prognostic implications. Journal of the American College of Cardiology, v. 51, n. 18, p. 1725-1733, 6 maio 2008.

LUPPINO, F. S. et al. Overweight, obesity, and depression: a systematic review and meta-analysis of longitudinal studies. Archives of General Psychiatry, v. 67, n. 3, p. 220-229, mar. 2010. 
Artigo Publicado no Vol.06 N.16 - Edição Especial 2020 - ISSN 2359-4829

Versão on-line disponível em: http://www.revistaflammae.com

MALIK, M. et al. CrossTalk Proposal: Heart Rate Variability Is a Valid Measure of Cardiac Autonomic Responsiveness. The Journal of physiology, v. 597, n. 10, maio 2019.

MCLEOD, J. C.; STOKES, T.; PHILLIPS, S. M. Resistance Exercise Training as a Primary Countermeasure to Age-Related Chronic Disease. Frontiers in Physiology, v. 10, 6 jun. 2019.

MORRIS, J. N. et al. Coronary heart-disease and physical activity of work. Lancet (London, England), v. 265, n. 6795, p. 1053-1057; contd, 21 nov. 1953.

NARCISO, F. V. et al. Effects of Shift Work on the Postural and Psychomotor Performance of Night Workers. PLOS ONE, v. 11, n. 4, p. e0151609, 26 abr. 2016.

NATIONAL FIRE PROTECTION ASSOCIATION. NFPA 1852: Standard on Selection, Care, and Maintenance of Open-Circuit Self-Contained Breathing Apparatus (SCBA). Disponível em: <https://www.nfpa.org/codesand-standards/all-codes-and-standards/list-of-codes-and-

standards/detail?code=1852>. Acesso em: 15 jun. 2020.

NFPA History. Disponível em: <https://www.nfpa.org/About-NFPA/NFPAoverview/History-of-NFPA>. Acesso em: 9 jun. 2020.

NOGUEIRA, E. C. et al. Body Composition is Strongly Associated With Cardiorespiratory Fitness in a Large Brazilian Military Firefighter Cohort: The Brazilian Firefighters Study. Journal of Strength and Conditioning Research / National Strength \& Conditioning Association, v. 30, n. 1, p. 33-38, jan. 2016.

NOGUEIRA, R. M.Avaliação da função autonômica cardíaca e da sobrecarga cardiovascular de bombeiros militares durante turno de serviço operacional. Dissertação / Dissertation-Faculdade de Educação Física: Universidade de Brasília. Orientador: Luiz Guilherme Grossi Porto, 16dez. 2016.

PATE, R. R. et al. Physical activity and public health. A recommendation from the Centers for Disease Control and Prevention and the American College of Sports Medicine. JAMA, v. 273, n. 5, p. 402-407, 1 fev. 1995.

PHELPS, S. M. et al. Characteristics and Predictors of Occupational Injury Among Career Firefighters. Workplace Health \& Safety, v. 66, n. 6, p. 291301, jun. 2018.

PHYSICAL ACTIVITY GUIDELINES ADVISORY COMMITTEE. Physical activity guidelines advisory committee scientific report. Washington, DC: US Department of Health and Human Services, 2018. 
Artigo Publicado no Vol.06 N.16 - Edição Especial 2020 - ISSN 2359-4829

Versão on-line disponível em: http://www.revistaflammae.com

PORTO, L. G. G. Risco cardiometabólico da atividade de bombeiro: estratégais individuais e institucionais na redução do risco e na promoção da saúde, com destaque para o papel da aptidão física. Revista FLAMMAE, v. 4, n. 11, p. 9, dez. 2018.

PORTO, L. G. G. et al. Agreement between BMl and body fat obesity definitions in a physically active population. Archives of Endocrinology and Metabolism, v. 60 , n. 6 , p. 515-525, dez. 2016.

PORTO, L. G. G. et al. Firefighters' basal cardiac autonomic function and its associations with cardiorespiratory fitness. Work (Reading, Mass.), v. 62, n. 3, p. 485-495, 2019a.

PORTO, L. G. G. et al. Firefighters' Quality of Life is Positively Associated With Cardiorespiratory Fitness Both on Mem and Women: 1027: Board \#261 May 29 2:00 PM - 3:30 PM. Medicine \& Science in Sports \& Exercise, v. 51, n. 6, p. 271-272, jun. 2019b.

POSTON, W. S. C. et al. Obesity and injury-related absenteeism in a population-based firefighter cohort. Obesity (Silver Spring, Md.), v. 19, n. 10, p. 2076-2081, out. 2011.

PUHL, R. M.; HEUER, C. A. Obesity stigma: important considerations for public health. American Journal of Public Health, v. 100, n. 6, p. 1019-1028, jun. 2010.

QIU, S. et al. Heart Rate Recovery and Risk of Cardiovascular Events and AllCause Mortality: A Meta-Analysis of Prospective Cohort Studies. Journal of the American Heart Association, v. 6, n. 5, 9 maio 2017.

REES-PUNIA, E. et al. Mortality Risk Reductions for Replacing Sedentary Time With Physical Activities. American Journal of Preventive Medicine, v. 56, n. 5, p. 736-741, maio 2019.

RHEA, M. R.; ALVAR, B. A.; GRAY, R. Physical fitness and job performance of firefighters. Journal of Strength and Conditioning Research, v. 18, n. 2, p. 348-352, maio 2004.

RIBEIRO, M. et al. Prevalence and risk of asthma symptoms among firefighters in São Paulo, Brazil: a population-based study. American Journal of Industrial Medicine, v. 52, n. 3, p. 261-269, mar. 2009.

SAARI, A. HEART RATE DYNAMICS DURING AND AFTER SIMULATED FIRE GROUND TASKS: EFFECTS OF PHYSICAL FITNESS AND TRAINING. Theses and Dissertations--Kinesiology and Health Promotion, 1 jan. 2019.

SAINT MARTIN, D. Avaliação objetiva do nível de atividade física, do comportamento sedentário e da aptidão cardiorrespiratória de bombeiros 
Artigo Publicado no Vol.06 N.16 - Edição Especial 2020 - ISSN 2359-4829

Versão on-line disponível em: http://www.revistaflammae.com

militares. Dissertação / Dissertation-Faculdade de Educação Física: Universidade de Brasília. Orientador: Luiz Guilherme Grossi Porto, 3 ago. 2018.

SAINT MARTIN, D. et al. Atividade física e comportamento sedentário de bombeiros em escala de $24 \mathrm{~h}$ trabalho por $72 \mathrm{~h}$ de folga. Revista Brasileira de Atividade Física \& Saúde, v. 24, n. Suppl 1, p. 228, 2019.

SANTOS, L. A expectativa de sobrevida do bombeiro militar do DF e a reforma dos regimes de Previdência Pública Brasileira. Brasília, DF: Corpo de Bombeiros Militar do Distrito Federal -Monografia - Curso de Altos Estudos para Oficiais, 2011.

SEGEDI, L. C. Análise da qualidade de vida, do nível de atividade física, da aptidão física e de fatores associados em bombeiros militares de ambos os gêneros. Dissertação / Dissertation-Faculdade de Educação Física: Universidade de Brasília, Orientador: Luiz Guilherme Grossi Porto.2 ago. 2018.

SMITH, D. L. et al. Cardiovascular Strain of Firefighting and the Risk of Sudden Cardiac Events: Exercise and Sport Sciences Reviews, v. 44, n. 3, p. 90-97, jul. 2016.

SMITH, D. L. et al. Pathoanatomic Findings Associated With Duty-Related Cardiac Death in US Firefighters: A Case-Control Study. Journal of the American Heart Association, v. 7, n. 18, p. e009446, 18 set. 2018.

SMITH, D. L. et al. The Relation of Emergency Duties to Cardiac Death Among US Firefighters. The American Journal of Cardiology, v. 123, n. 5, p. 736741, 012019.

SOTERIADES, E. S. et al. Cardiovascular disease in US firefighters: a systematic review. Cardiology in Review, v. 19, n. 4, p. 202-215, ago. 2011.

SOTERIADES, E. S. et al. Cancer Incidence and Mortality in Firefighters: A State-of-the-Art Review and Meta-Analysis. Asian Pacific Journal of Cancer Prevention : APJCP, v. 20, n. 11, p. 3221-3231, 2019.

STENHOLM, S. et al. Long-Term Determinants of Muscle Strength Decline: Prospective Evidence from the 22-Year Mini-Finland Follow-Up Survey. Journal of the American Geriatrics Society, v. 60, n. 1, p. 77-85, 2012.

SULLIVAN, J. P. et al. Randomized, Prospective Study of the Impact of a Sleep Health Program on Firefighter Injury and Disability. Sleep, v. 40, n. 1, jan. 2017.

THE IMAGE STUDY GROUP et al. Systematic review of reviews of intervention components associated with increased effectiveness in dietary and physical activity interventions. BMC Public Health, v. 11, n. 1, p. 119, dez. 2011. 
THOMPSON, W. R. et al. ACSM's Guidelines for Exercise Testing and Prescription. 10. ed. Philadelphia: Lippincott Williams \& Wilkins, 2018.

TIELAND, M.; TROUWBORST, I.; CLARK, B. C. Skeletal muscle performance and ageing. Journal of Cachexia, Sarcopenia and Muscle, v. 9, n. 1, p. 3-19, 2018.

WATKINS, E. R. et al. Women Firefighters' Health and Well-Being: An International Survey. Women's Health Issues: Official Publication of the Jacobs Institute of Women's Health, v. 29, n. 5, p. 424-431, out. 2019.

WILLIAMS-BELL, F. M. et al. Air management and physiological responses during simulated firefighting tasks in a high-rise structure. Applied Ergonomics, v. 41, n. 2, p. 251-259, mar. 2010.

WORLD HEALTH ORGANIZATION. Obesity and overweight - Key facts. Disponível em: <https://www.who.int/news-room/fact-sheets/detail/obesity-andoverweight>. Acesso em: 11 jun. 2020.

YAMAMOTO, $\mathrm{K}$. et al. Effects of endurance training on resting and postexercise cardiac autonomic control. Medicine and Science in Sports and Exercise, v. 33, n. 9, p. 1496-1502, set. 2001.

YANG, J. et al. Association Between Push-up Exercise Capacity and Future Cardiovascular Events Among Active Adult Men. JAMA Network Open, v. 2, n. 2, p. e188341-e188341, 1 fev. 2019. 